Article

\title{
Upper Limits to Magnetic Fields in the Outskirts of Galaxies
}

\author{
Ericson López *, Jairo Armijos-Abendaño, Mario Llerena and Franklin Aldás
}

Observatorio Astronómico de Quito, Escuela Politécnica Nacional, Av. Gran Colombia S/N, Quito 170403, Ecuador; jairo.armijos@epn.edu.ec (J.A.-A.); mario.llerena01@epn.edu.ec (M.L.); franklin.aldas@epn.edu.ec (F.A.)

* Correspondence: ericsson.lopez@epn.edu.ec

Academic Editor: Tomotsugu Goto

Received: 31 July 2017; Accepted: 18 September 2017; Published: 19 September 2017

\begin{abstract}
Based on $\mathrm{CO}(2-1)$ public data, we study the monoxide oxygen gas excitation conditions and the magnetic field strength of four spiral galaxies. For the galaxy outskirts, we found kinetic temperatures in the range of $\lesssim 35-38 \mathrm{~K}$, CO column densities $\lesssim 10^{15}-10^{16} \mathrm{~cm}^{-2}$, and $\mathrm{H}_{2}$ masses $\lesssim 4 \times 10^{6}-6 \times 10^{8} \mathrm{M}_{\odot}$. An $\mathrm{H}_{2}$ density $\lesssim 10^{3} \mathrm{~cm}^{-3}$ is suitable to explain the $2 \sigma$ upper limits of the $\mathrm{CO}(2-1)$ line intensity. We constrain the magnetic field strength for our sample of spiral galaxies and their outskirts by using their masses and $\mathrm{H}_{2}$ densities to evaluate a simplified magneto-hydrodynamic equation. Our estimations provide values for the magnetic field strength on the order of $\lesssim 6-31 \mu \mathrm{G}$.
\end{abstract}

Keywords: nearby galaxies; magnetic fields; molecules

\section{Introduction}

In this paper, we focus our attention on the study of the magnetic field strength in the outskirts of four spiral galaxies, following a different approach to those commonly based on Faraday rotation, dust polarization, synchrotron emission, etc. To constrain the magnetic field strength of spiral galaxies, we will follow the Dotson method, described in Section 4.4 of [1]; i.e., approaching the magneto-hydrodynamic force equation to derive a simple expression to estimate the upper limit of the magnetic field.

\section{Magnetic Field Constraint}

As mentioned above, we use the Dotson method [1] to constrain the magnetic field, which is mainly based on the following relation:

$$
B<3.23 \times 10^{-8}\left(\frac{R}{\mathrm{pc}}\right)^{0.5}\left(\frac{n}{\mathrm{~cm}^{-3}}\right)^{0.5}\left(\frac{M}{\mathrm{M}_{\odot}}\right)^{0.5}\left(\frac{r}{\mathrm{pc}}\right)^{-1}
$$

where $R$ is the radius of the magnetic field lines, $n$ is the molecular hydrogen gas density, $M$ and $r$ are the total mass and radius of the source, respectively. So, the magneto-hydrodynamic force can be used to estimate the magnetic field strength (Equation (1)) when there is available information about the shape of the field lines (see [1]). On the other hand, to estimate the density $n$ and mass $M$ of the source, we use the carbon monoxide emission as a tracer of the molecular gas $\mathrm{H}_{2}$ [2]. This is because $\mathrm{H}_{2}$ is invisible in the cold interstellar medium (around 10-20 K), so its distribution and motion must be inferred from observations of minor constituents of the clouds, such as carbon monoxide and dust [2]. Carbon monoxide is the most abundant molecule after $\mathrm{H}_{2}, \mathrm{CO}$ is easily excited, and the emission of $\mathrm{CO}(1-0)$ at $2.6 \mathrm{~mm}$ is ubiquitous in the Galaxy [2]. So, $\mathrm{CO}$ it is a good tracer for molecular hydrogen. 


\section{Carbon Monoxide Data}

To carry out this study, we used public $\mathrm{CO}(2-1)$ data, first published by [3], data obtained with the IRAM $30 \mathrm{~m}$ telescope ${ }^{1}$ located in Spain. At the $\mathrm{CO}(2-1)$ transition frequency $(230.538 \mathrm{GHz})$, the IRAM telescope has a spatial resolution of 13 arcsec. From the available data we selected a sample of four nearby spiral galaxies, whose morphology and positions are listed in Table 1. On the other hand, in Figure 1, CO(2-1) integrated intensity maps of each galaxy in our sample are shown.

Table 1. Galaxy sample morphology and positions.

\begin{tabular}{ccccc}
\hline Galaxy Name & $\begin{array}{c}\text { RA }^{\mathbf{1}} \\
\text { (hh:mm:ss.s) }\end{array}$ & $\begin{array}{c}\text { DEC }^{\mathbf{1}} \\
\text { (hh:mm:ss.s) }\end{array}$ & Morphology $^{\mathbf{1}}$ & $\begin{array}{c}\text { Distance }^{\mathbf{1}} \\
\text { (Mpc) }\end{array}$ \\
\hline NGC 2841 & 09:22:02.7 & $+50: 58: 35.3$ & SAa C & 14.6 \\
NGC 3077 & $10: 03: 19.1$ & $+68: 44: 02.2$ & S0 C & 3.8 \\
NGC 3184 & $10: 18: 17.0$ & $+41: 25: 27.8$ & SAc C & 11.3 \\
NGC 3351 & $10: 43: 57.7$ & $+11: 42: 13.0$ & SBb C & 10.5 \\
\hline
\end{tabular}

${ }^{1}$ Information taken from the SIMBAD Astronomical Database.
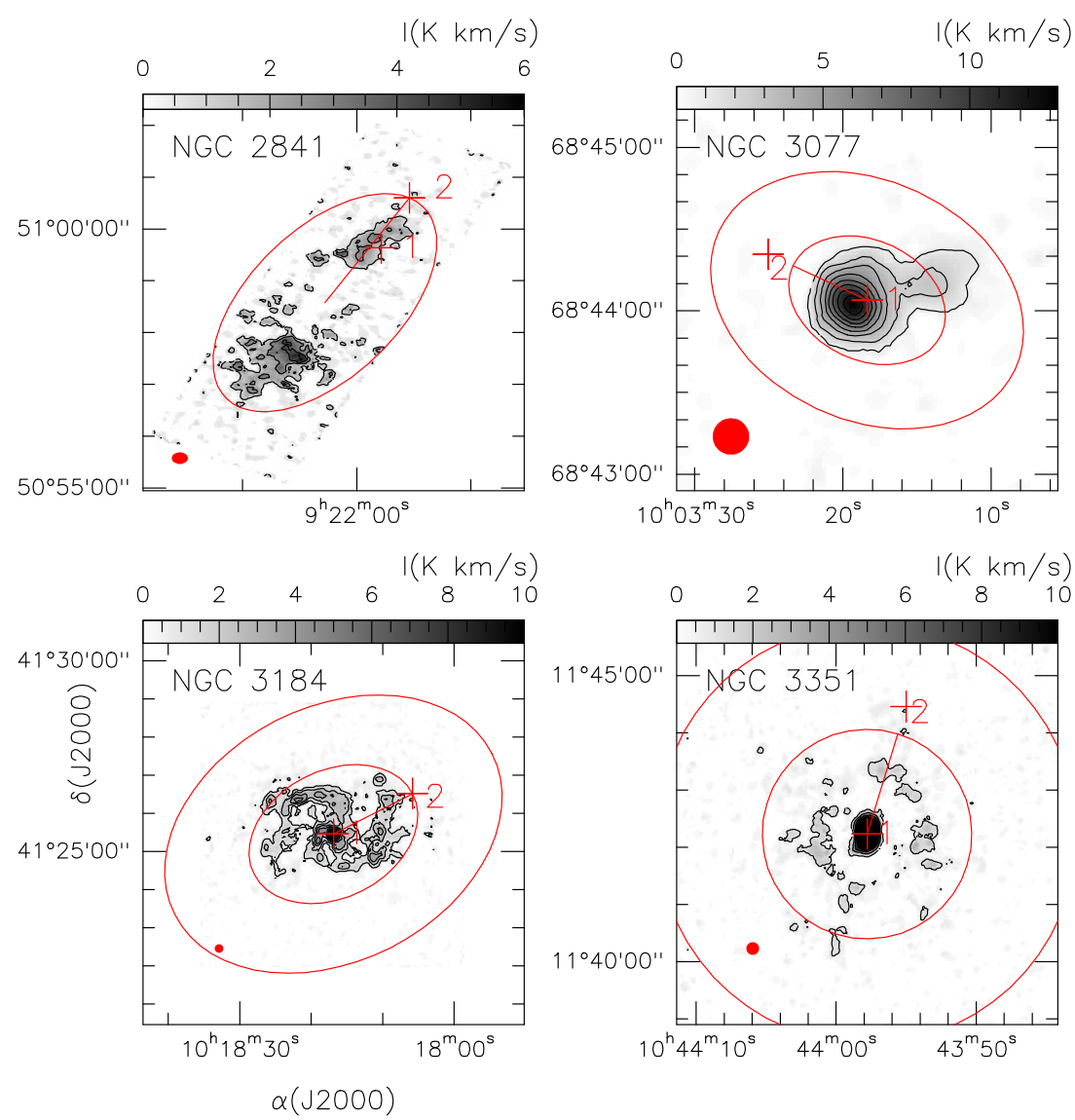

Figure 1. $\mathrm{CO}(2-1)$ integrated intensity maps of our galaxy sample. The red crosses show positions used to extract the spectra shown in Figure 2. The red ellipses indicate the regions used to measure $\mathrm{CO}(2-1)$ luminosities (see text). The tiny filled red ellipse represents the IRAM telescope beam (13 arcsec at the $\mathrm{CO}(2-1)$ frequency transition of $230.538 \mathrm{GHz})$. For NGC 2841 , we did not measure the $\mathrm{CO}(2-1)$ luminosity in the galaxy outskirts, as in this particular case was not observed.

1 http://www.iram-institute.org/EN/30-meter-telescope.php 


\section{Data Treatment}

\subsection{Spectra Selection}

As was mentioned in the introduction, in order to constrain the magnetic field strengths for the galaxies in our sample, we use the method applied by [1], which is based on Equation (1). Therefore, for every source, the parameters $R, n, M$, and $r$ should be determined or assumed. We estimate $n, M$, and $r$ from $\mathrm{CO}(2-1)$ observations (see Section 4), while $R$ is assumed based on the results of previous works [4-7].

To derive $n$ and $M$ from the $\mathrm{CO}(2-1)$ data we have selected the spectra by choosing two positions; one located in the nucleus of the galaxy (hereinafter the position 1) and the second located on the outskirts of the galaxy (hereinafter the position 2). $\mathrm{CO}(2-1)$ line emission is not detected towards the NGC 2841 nucleus; therefore, as an exception, its position 1 spectrum corresponds to a position displaced 79 arcsec from the galaxy nucleus. The inner ellipse is taken as the one that holds as much as possible the integrated intensity of $\mathrm{CO}(2-1)$ radiation emitted by the galaxy. The center of this ellipse defines the nucleus (position 1). Starting from the nuclei, in steps of 20 arcsec, we take $\mathrm{CO}(2-1)$ spectrum along the major axis of the inner ellipse. Position 2 is defined as the more contiguous positions to the galaxy nucleus, placed along the galaxy major-axis, where the $\mathrm{CO}(2-1)$ line emission is no longer detected above $2 \sigma$. We assume that position 2 is representative of the lowest boundary of the galactic outskirts which are well traced by the HI emission [8]. In Figure 1, positions 1 and 2 are indicated by red crosses. For all galaxies in our sample, the spectra extracted for both positions, within the 13 arcsec resolution of the IRAM telescope, are shown in Figure 2. In our study, the choice of position 2 depends on two aspects: (1) the IRAM telescope beam size and (2) the step of 20 arcsec used to find one of the nearest position to the galaxy nucleus, along the major-axis, where $\mathrm{CO}(2-1)$ emission is no longer detected.
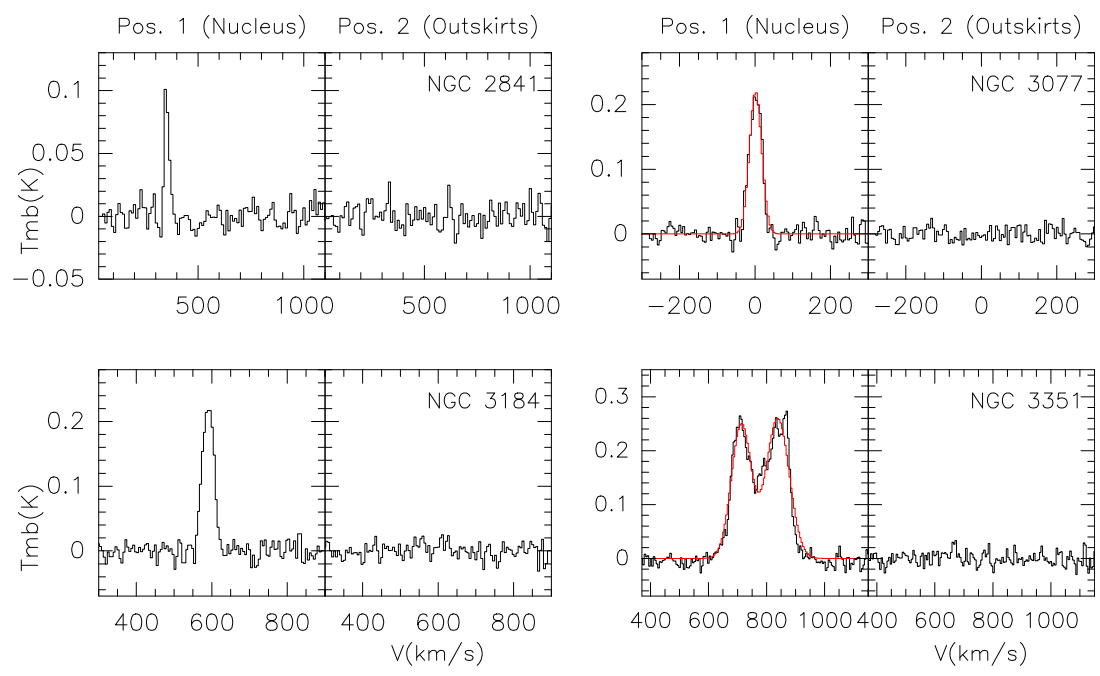

Figure 2. Spectra extracted from positions 1 and 2 indicated in Figure 1. As an example, for NGC 3077 and NGC 3351 galaxies, we show the Gaussian fits (indicated with red curves) applied to the $\mathrm{CO}(2-1)$ lines.

\subsection{Gaussian Fitting and $\mathrm{CO}$ Luminosities}

Gaussian fits to the $\mathrm{CO}(2-1)$ lines (indicated in Figure 2) have been performed. Then, the large velocity gradient (LVG) modeling [9] is employed to estimate the gas density $(n)$, based on the $\mathrm{CO}(2-1)$ line width $(\Delta V)$ and the line intensity $(I)$. For position 1 (nucleus) we derived the values of these parameters, which are presented in Table 2 . For position 2 (outskirts), we estimated $2 \sigma$ upper limits for the $\mathrm{CO}(2-1)$ line intensity, which are also given in Table 2. This table includes the radial velocity $(V)$ and the average $\overline{\Delta V}$ obtained from spectra extracted along the major-axis of the galaxy disk. 
To estimate $\mathrm{CO}$ integrated luminosities $\left(L_{\mathrm{CO}}\right)$ for the galaxies in our sample, we used the inner ellipse for the nuclear region (the $L_{C O}$ will be used later to estimate $\mathrm{H}_{2}$ masses). As seen in Figure 1 , and as it was indicated before, this ellipse is defined to enclose almost all the $\mathrm{CO}(2-1)$ emission arising from the galaxy disk. In turn, to estimate a $2 \sigma$ upper limit on the $L_{C O}$ for the galaxy outskirts, we use a ringlike region with axes equal to twice the sizes of the inner ellipse. The derived $L_{C O}$ values for both regions of each galaxy are given in Table 3.

Table 2. $\mathrm{CO}(2-1)$ line properties derived for our galaxy sample.

\begin{tabular}{|c|c|c|c|c|c|}
\hline \multirow{2}{*}{ Galaxy Name } & \multirow{2}{*}{ Region } & $\Delta V(\sigma)$ & $V(\sigma)$ & $I^{(2)}$ & $\overline{\Delta V}^{(3)}$ \\
\hline & & $\mathrm{km} \mathrm{s}^{-1}$ & $\mathrm{~km} \mathrm{~s}^{-1}$ & $\mathbf{K}$ & $\mathrm{km} \mathrm{s}^{-1}$ \\
\hline \multirow{2}{*}{ NGC 2841} & $\begin{array}{l}\text { Position displaced } 79 \text { arcsec } \\
\text { from nucleus }\end{array}$ & $25.2(2.0)$ & 352.0 & 0.1 & 23.4 \\
\hline & outskirts & $\ldots$ & $\ldots$ & $\lesssim 0.03$ & $\ldots$ \\
\hline \multirow{2}{*}{ NGC 3077} & nucleus & $38.2(1.1)$ & $1.8(0.5)$ & 0.2 & 40.2 \\
\hline & outskirts & $\ldots$ & $\ldots$ & $\lesssim 0.02$ & $\ldots$ \\
\hline \multirow{2}{*}{ NGC 3184} & nucleus & $35.4(1.0)$ & $590.3(0.5)$ & 0.2 & 25.6 \\
\hline & outskirts & $\ldots$ & $\ldots$ & $\lesssim 0.03$ & $\ldots$ \\
\hline \multirow{2}{*}{ NGC 3351} & nucleus & $80.9(2.3)$ & $713.1(0.9)$ & 0.3 & 45.9 \\
\hline & outskirts & $\ldots$ & $\ldots$ & $\lesssim 0.03$ & $\ldots$ \\
\hline
\end{tabular}

(1) $\mathrm{As} \mathrm{CO}(2-1)$ line emission is not detected at the galaxy nucleus, this position is displaced 79 arcsec from the galaxy nucleus (see Figure 1); ${ }^{(2)}$ For galaxy outskirts, this parameter is a $2 \sigma$ upper limit based on the rms noise; ${ }^{(3)}$ Average value obtained from several spectra extracted along the major-axis of the galaxy disk.

Table 3. Parameters derived for the galaxy sample.

\begin{tabular}{|c|c|c|c|c|c|c|}
\hline \multirow{2}{*}{ Galaxy Name } & \multirow{2}{*}{ Region } & $L_{C O}{ }^{(2)}$ & $r^{(3)}$ & $M_{H_{2}}(4)$ & $\left(M_{H_{2}}+M_{H I}\right)^{(5)}$ & B \\
\hline & & $\times 10^{6} \mathrm{~K} \mathrm{~km} \mathrm{~s}^{-1} \mathrm{pc}^{2}$ & kpc & $\times 10^{7} \mathbf{M}_{\odot}$ & $\times 10^{8} M_{\odot}$ & $\mu \mathrm{G}$ \\
\hline \multirow{2}{*}{ NGC 2841} & \multirow{2}{*}{$\begin{array}{c}\text { disk } \\
\text { outskirts }\end{array}$} & 2.0 & 8.5 & 103.0 & 89.6 & $\lesssim 31$ \\
\hline & & $\cdots$ & $\cdots$ & $\ldots$ & $\ldots$ & $\cdots$ \\
\hline \multirow{2}{*}{ NGC 3077} & disk & 2.3 & 0.4 & 1.3 & 0.4 & $\lesssim 6$ \\
\hline & outskirts & $\lesssim 0.7$ & 0.8 & $\lesssim 0.4$ & $\lesssim 0.1$ & $\lesssim 7$ \\
\hline \multirow{2}{*}{ NGC 3184} & disk & 188.0 & 6.6 & 103.0 & 36.8 & $\lesssim 14$ \\
\hline & outskirts & $\lesssim 81.1$ & 13.2 & $\lesssim 44.6$ & $\lesssim 15.9$ & $\lesssim 19$ \\
\hline \multirow{2}{*}{ NGC 3351} & disk & 226.0 & 7.1 & 124.0 & 32.1 & $\lesssim 11$ \\
\hline & outskirts & $\lesssim 109.0$ & 14.2 & $\lesssim 59.8$ & $\lesssim 15.5$ & $\lesssim 15$ \\
\hline
\end{tabular}

(1) For NGC 2841 we were not able to estimate $L_{\mathrm{CO}}$ luminosity and other relative parameters in the outskirts, as this region was not observed; (2),(4),(5) For galaxy outskirts, these parameters are a $2 \sigma$ upper limit;

(3) This radius traces the boundary of the galaxy disk region, where the $\mathrm{CO}(2-1)$ line emission is still detectable above $2 \sigma$ (see Section 3.1).

\section{Results}

\subsection{Galaxy Mass}

The molecular hydrogen mass $\left(M_{H_{2}}\right)$ for the galaxy disk and its outskirts is derived using the the equation given by [3]:

$$
\frac{M_{H_{2}}}{\mathrm{M}_{\odot}}=5.5 \frac{R_{21}}{0.8}\left(\frac{L_{C O}}{\mathrm{Kkm} \mathrm{s}^{-1} \mathrm{pc}^{2}}\right)
$$

where $R_{21}$ is the $\mathrm{CO}(2-1) / \mathrm{CO}(1-0)$ line intensity ratio (equal to 0.8$)$ and $L_{\mathrm{CO}}$ is the $\mathrm{CO}$ luminosity. In this equation, a $\mathrm{CO}(1-0) / \mathrm{H}_{2}$ conversion factor of $2 \times 10^{20} \mathrm{~cm}^{-1}\left(\mathrm{~K} \mathrm{~km} \mathrm{~s}^{-1}\right)^{-1}$ is adopted [3]. In Table 3, the $M_{H_{2}}$ values are listed for both galaxy regions. We found that the $\mathrm{H}_{2}$ outskirts masses derived for 
our galaxies sample vary within $\lesssim 4 \times 10^{6}-1 \times 10^{9} \mathrm{M}_{\odot}$. The total mass $\left(M_{H_{2}}+M_{H I}\right)$ is also presented in the same table. The atomic hydrogen mass has been estimated by using the $M_{H_{2}} / M_{H I}$ ratio given in [3].

\section{2. $\mathrm{H}_{2}$ Density, CO Column Density, and Kinetic Temperature}

As was mentioned above, the $\mathrm{H}_{2}$ density is derived for the nucleus and outskirts positions in the galaxies of our sample by using the LVG approach. We fit the $\mathrm{CO}(2-1)$ line intensity or its limit and the average line width $\overline{\Delta V}$, while the $\mathrm{CO}$ column density $\left(N_{\mathrm{CO}}\right), \mathrm{H}_{2}$ molecular density $\left(n_{\mathrm{H}_{2}}\right)$, and the kinetic temperature $\left(T_{k i n}\right)$ are considered as free parameters. The $\mathrm{CO}(1-0)$ line intensity is known based on the $\mathrm{CO}(2-1) / \mathrm{CO}(1-0)$ line intensity ratio of about 0.8 derived by [3]. Note that at position 2 (i.e., outskirt positions), we do not detect $\mathrm{CO}(2-1)$ emission and we have derived $2 \sigma$ upper limits for these line intensities. We found that a $n_{\mathrm{H}_{2}}$ density of about $10^{3} \mathrm{~cm}^{-3}$ is suitable to fit the $\mathrm{CO}(2-1)$ line intensity for the four galaxies in our sample. For positions 1 and 2, the $N_{C O}$ and $T_{k i n}$ that provide the best fits to the $\mathrm{CO}(2-1)$ line intensity (or its limit) are given in Table 4. For the galaxy outskirts, we found $T_{k i n} \lesssim 35-38 \mathrm{~K}$ and $N_{C O} \lesssim 10^{15}-10^{16} \mathrm{~cm}^{-2}$. These findings tell us that the molecular gas in galaxy outskirts is relatively cold. For the nucleus positions, the $T_{k i n}$ are found within $35-38 \mathrm{~K}$ and the $N_{C O}$ within $\sim 10^{15}-10^{16} \mathrm{~cm}^{-2}$. So, the column density $N_{C O}$ exhibits the greatest changes, whereas the kinetic temperature $T_{k i n}$ is relatively constant. The physical parameters presented in Table 4 seem to be similar for both nuclei and outskirts, but in the case of the nuclei the given values are accurate, while for the outskirts they correspond to the $2 \sigma$ upper limits.

Table 4. Physical parameters derived for our galaxy sample.

\begin{tabular}{cccc}
\hline Component & $n_{\boldsymbol{H}_{2}}$ & $T_{\text {kin }}$ & $N_{C O}$ \\
\hline outskirts & $\lesssim 10^{3} \mathrm{~cm}^{-3}$ & $\lesssim 35-38 \mathrm{~K}$ & $\lesssim 10^{15}-10^{16} \mathrm{~cm}^{-2}$ \\
nucleus & $10^{3}$ & $35-38 \mathrm{~K}$ & $\sim 10^{15}-10^{16} \mathrm{~cm}^{-2}$ \\
\hline
\end{tabular}

\subsection{Magnetic Fields in Galaxies and Their Outskirts}

As mentioned previously, in order to constrain the magnetic field strength for a given galaxy and its outskirts, we use Equation (1) given by [1]. This equation includes the $n_{H_{2}}$, the mass $M$, and radius $r$ of the galaxy, and the radius of curvature $R$ of the magnetic field lines. $n_{H_{2}}$ and $M$ were derived in the previous sections, but the mass that we use in Equation (1) refers to the dust mass, which is obtained by the relation $\left(M_{H_{2}}+M_{H I}\right) / 100$; i.e., assuming the typical dust-to-gas mass ratio of 0.01 . In this section, we assume that $R$ is equal to the radius $r$ of the studied regions. This assumption is crude, but it is based on several studies of spiral galaxies such as NGC 4736, M51, NGC 1097, and NGC 1365, which reveal magnetic field lines that extend as far as their galactic arms [4-6]. The derived values for the magnetic field strength are listed in Table 3. For the galaxy outskirts, we have considered the upper limits of $M$ and $n$ as fixed values in order to calculate the magnetic field strength. For the NGC 2841 galaxy, no value was estimated for the magnetic field in its outskirts, because this region was not observed in this object. We found magnetic field magnitudes on the order of $\lesssim 6-31 \mu \mathrm{G}$ for the galaxies in our sample and their outskirts. These limits agree with those values of $\sim 20-60 \mu \mathrm{G}$ found in spiral galaxies $[4,6,7]$.

\section{Conclusions}

In the present contribution, we have estimated the magnetic field strength in the galaxy nuclei and in the outskirts of a sample of four spiral galaxies. For that, we have used an approximate expression of the magnetohydrodynamics to find an upper limit for the magnetic field magnitudes. The magnetic field strength lies within the range of $\lesssim 6-31 \mu \mathrm{G}$, which is in good agreement with the values provided by other authors for spiral galaxies. A first good idea about the strength of the magnetic field can be 
obtained directly from the estimation of molecular hydrogen mass and radio of the source, without the necessity of a magnetohydrodynamical model or the use of a traditional technique like Faraday rotation or Zeeman line broadening. This is a rough estimation that works if the gas pressure is uniform and the viscosity is neglected. A better approach can be obtained by keeping more terms in the magnetohydrodynamics force equation [1] to impede gravitational collapse.

Moreover, instead of using the total hydrogen mass $\left(M_{H_{2}}+M_{H I}\right)$ in our magnetic field calculations, the mass of the dust has been considered as enough to get values in good agreement with the $\sim 20-60 \mu \mathrm{G}$ observed in spiral galaxies $[4,6,7]$. This fact suggests that the dust is the main component that influences the magnetic field strength better than the molecular gas.

On the other hand, for the galaxy outskirts we found a kinetic temperature $T_{k i n}$ of about $\lesssim 35-38 \mathrm{~K}$ and a column density $N_{C O} \lesssim 10^{15}-10^{16} \mathrm{~cm}^{-2}$. These findings tell us that the molecular gas in galaxy outskirts is relatively cold. Moreover, the $M_{\mathrm{H}_{2}}$ masses on the outskirts of the galaxy are in the range of $\lesssim 4 \times 10^{6}-6 \times 10^{8} \mathrm{M}_{\odot}$, and a $n_{\mathrm{H}_{2}}$ density of $\lesssim 10^{3} \mathrm{~cm}^{-3}$ is suitable to explain the $2 \sigma$ upper limits to the $\mathrm{CO}(2-1)$ line intensity. Then, it seems that if the densities and temperatures are low in the outskirts it would result in a higher $M_{H_{2}}$ mass at a given $\mathrm{CO}(2-1)$ luminosity if the outskirts volume is increasing. It is interesting that the values of both $\mathrm{H}_{2}$ density and kinetic temperature are relatively similar in the nuclear region of the studied galaxies, but not the $\mathrm{CO}$ column density that varies by a factor of 10 along our sample.

In future research, we plan to go deeper in understanding the magnetic field structure in galaxy halos, studying more spiral galaxies and employing other molecular hydrogen tracers. Additionally, understanding the variations of the field direction associated with the column density changes is part of our future work.

Author Contributions: E. López, J. Armijos-Abendaño, M. Llerena and F. Aldás performed the data analysis. E. López and J. Armijos-Abendaño wrote the manuscript. All authors contributed to the discussion and interpretation of the results.

Conflicts of Interest: The authors declare no conflict of interest.

\section{References}

1. Dotson, J.L. Polarization of the Far-Infrared Emission from M17. Astrophys. J. 1996, 470, 566-576.

2. Neininger, N.; Guélin, M.; Ungerechts, H.; Lucas, R.; Wielebinski, R. Carbon monoxide emission as a precise tracer of molecular gas in the Andromeda galaxy. Nature 1998, 395, 871-873.

3. Leroy, A.K.; Walter, F.; Bigiel, F.; Usero, A.; Weiss, A.; Brinks, E.; de Blok, W.J.G.; Kennicutt, R.C.; Schuster, K.F.; Kramer, C.; et al. Heracles: The HERA CO Line Extragalactic Survey. Astrophys. J. 2009, 137, 4670-4696.

4. Beck, R.; Fletcher, A.; Shukurov, A.; Snodin, A.; Sokoloff, D.D.; Ehle, M.; Moss, D.; Shoutenkov, V. Magnetic fields in barred galaxies. IV. NGC 1097 and NGC 1365. Astron. Astrophys. 2005, 444, 739-765.

5. Chyzy, K.; Buta, R. Discovery of a Strong Spiral Magnetic Field Crossing the Inner Pseudoring of NGC 4736. Astrophys. J. Lett. 2008, 677, L17.

6. Fletcher, A.; Beck, R.; Shukurov, A.; Berkhuijsen, E.M.; Horellou, C. Magnetic fields and spiral arms in the galaxy M51. Mon. Not. R. Astron. Soc. 2011, 412, 2396-2416.

7. Knapik, J.; Soida, M.; Dettmar, R.; Beck, R.; Urbanik, M. Detection of spiral magnetic fields in two flocculent galaxies. Astron. Astrophys. 2000, 362, 910-920.

8. Sofue, Y. The most completely sampled rotation curves for galaxies. Astrophys. J. 1996, 458, 120-131.

9. Van der Tak, F.F.S.; Black, J.H.; Schöier, F.L.; Jansen, D.J.; van Dishoeck, E.F. A computer program for fast non-LTE analysis of interstellar line spectra. With diagnostic plots to interpret observed line intensity ratios. Astron. Astrophys. 2007, 468, 627-635.

(C) 2016 by the authors. Licensee MDPI, Basel, Switzerland. This article is an open access article distributed under the terms and conditions of the Creative Commons Attribution (CC BY) license (http:// creativecommons.org/licenses/by/4.0/). 